DOI: $10.15593 / 2224-9982 / 2018.55 .01$

УДК 519.9, 629.7

\author{
М.Ю. Егоров ${ }^{1}$, Д.М. Егоров ${ }^{2}$, С.М. Егоров ${ }^{2}$, А.А. Савочкина ${ }^{1}$ \\ ${ }^{1}$ Пермский национальный исследовательский политехнический университет, Пермь, Россия \\ ${ }^{2}$ Научно-исследовательский институт полимерных материалов, Пермь, Россия \\ ЧИСЛЕННОЕ ИССЛЕДОВАНИЕ ДИНАМИКИ ВНУТРИКАМЕРНЫХ \\ ПРОЦЕССОВ РАКЕТНОГО ДВИГАТЕЛЯ НА ТВЕРДОМ ТОПЛИВЕ ОСОБОЙ \\ КОМПОНОВОЧНОЙ СХЕМЫ. ЧАСТЬ 2. РЕЗУЛЬТАТЫ РАСЧЕТОВ
}

\begin{abstract}
Современный ракетный двигатель на твердом топливе - сложная техническая система, в которой одновременно протекает ряд взаимосвязанных нестационарных и нелинейных физико-химических процессов. Приводятся результаты численных расчетов динамики внутрикамерных процессов при срабатывании ракетного двигателя особой компоновочной схемы, полученные с использованием разработанной ранее физико-математической модели и созданного на ее основе пакета прикладных программ. При срабатывании воспламенительного устройства в камере сгорания наблюдается кратковременный локальный интенсивный всплеск давления. Далее по времени работы в камере сгорания, газоходах и сопловых блоках отслеживается затухающий волновой процесс с постепенным подъемом уровня давления за счет также постепенного прогрева и зажигания поверхности горения заряда твердого топлива. При достижении уровня давления, соответствующего некоторому критическому значению, вскрываются заглушки сопловых блоков. Давление в этот момент в камере сгорания резко падает. За счет интенсивного зажигания поверхности горения заряда твердого топлива давление в камере сгорания резко возрастает и достигает уровня рабочего значения. На этом этапе в боковых газоходах генерируется высокочастотный колебательный процесс. В целом в расчетах четко отслеживается сложный переходный волновой, асимметричный по пространству и переменный по времени характер течения в камере сгорания, газоходах и сопловом блоке. Для оптимизации параметров ракетного двигателя в работе рассматриваются три варианта его компоновочной схемы. В исходном варианте наблюдается значительная задержка зажигания поверхности горения заряда твердого топлива и, как следствие, затянутый по времени выход на расчетный режим работы. В двух оптимизированных вариантах задержка зажигания существенно уменьшается.

Ключевые слова: ракетный двигатель на твердом топливе, внутрикамерные процессы, горение, газовая динамика, результаты расчетов.
\end{abstract}

\author{
M.Yu. Egorov1, D.M. Egorov², S.M. Egorov², A.A. Savochkina1 \\ ${ }^{1}$ Perm National Research Polytechnic University, Perm, Russian Federation \\ ${ }^{2}$ Research Institute of Polymeric Materials, Perm, Russian Federation
}

\title{
NUMERICAL STUDY OF THE DYNAMICS OF INTRA-CHAMBER PROCESSES ROCKET ENGINE SOLID FUEL THE SPECIAL LAYOUT SCHEME. PART 2. RESULTS OF PAYMENTS
}

\begin{abstract}
A modern solid-fuel rocket engine is a complex technical system in which a number of interrelated non-stationary and nonlinear physical and chemical processes occur simultaneously. The article presents the results of numerical calculations of the dynamics of in-chamber processes in the operation of the rocket engine of a special layout scheme, obtained using the previously developed physical and mathematical model and created on its basis a package of applications. When the ignition device is activated, a short-term local intense pressure surge is observed in the combustion chamber. Further, by the time of operation in the combustion chamber, flues and nozzle blocks, a damped wave process is monitored with a gradual rise in the pressure level due to the gradual heating and ignition of the combustion surface of the solid fuel charge. When the pressure level corresponding to a certain critical value is reached, the plugs of the nozzle blocks are opened. The pressure at this point in the combustion chamber drops sharply. Further in time, due to the intensive ignition of the combustion surface of the solid fuel charge, the pressure in the combustion chamber increases sharply and reaches the level of the operating value. At this stage, a high-frequency oscillatory process is generated in the lateral flues. In General, the calculations clearly track the complex transient wave, space-asymmetric and time-variable nature of the flow in the combustion chamber, flues and nozzle block. To optimize the parameters of the rocket engine, three variants of its layout scheme are considered. In the original version there was a considerable delay of the ignition of burning surface of solid propellant charge and, as a consequence, tight on time output on the current mode of operation. In the two optimized variants, the ignition delay is significantly reduced.
\end{abstract}

Keywords: solid propellant rocket motors, intrachamber processes, combustion, gas dynamics, results of payments. 
Современный ракетный двигатель на твердом топливе (РДТТ) - сложная техническая система, в которой одновременно протекает ряд взаимосвязанных (сопряженных) нестационарных и существенно нелинейных физико-химических процессов [1-14 и др.]. Рассматриваемый РДТТ имеет свои конструктивные и функциональные особенности. Проанализируем некоторые из них детально.

Приведем результаты численных расчетов динамики внутрикамерных процессов при срабатывании данного ракетного двигателя, полученные с использованием разработанной ранее физико-математической модели (уровня постановки вычислительного эксперимента) и созданного на ее основе пакета прикладных программ [15]. Общий вид РДТТ особой компоновочной схемы показан на рис. 1. Все приведенные ниже функциональные параметры даны в безразмерном виде.

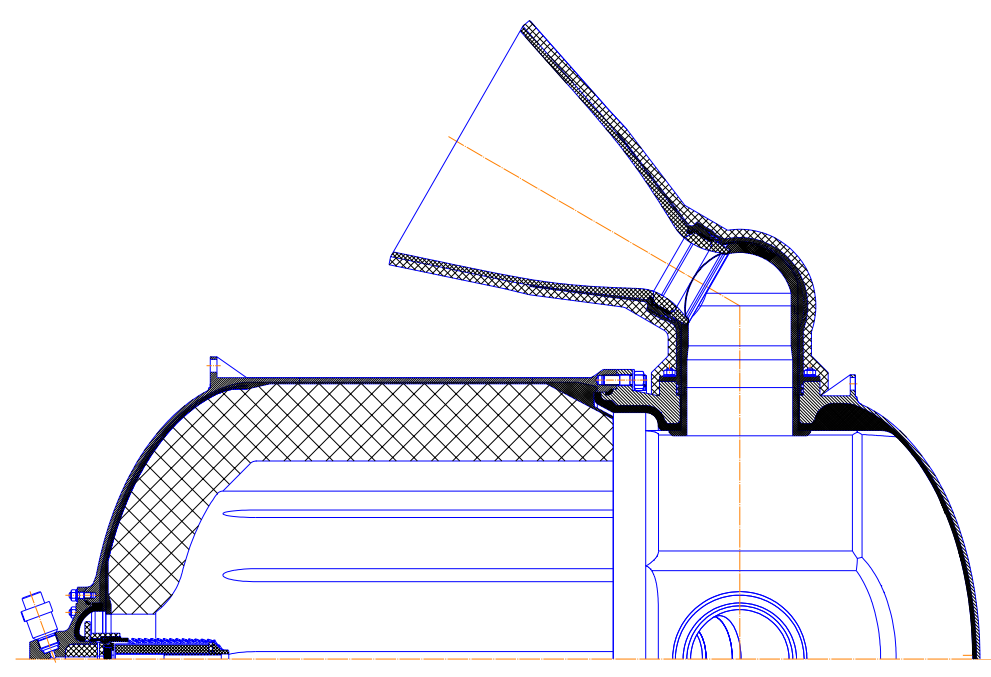

Рис. 1. РДТТ особой компоновочной схемы

На рис. 2-5 показано изменение во времени (до выхода на основной режим работы) давления продуктов сгорания (точнее, газовой смеси воздуха, продуктов сгорания воспламенительного состава и твердого топлива) в фиксированных точках газодинамического тракта РДТТ. Принятые обозначения: $P_{1}-$ давление в камере сгорания ракетного двигателя, $P_{2}-$ давление в вершине бокового газохода, $(-x)$ - крайняя отрицательная координата по оси $O X,(+x)-$ крайняя положительная координата по оси $O X,(-z)$ - крайняя отрицательная координата по оси $O Z(O Z$ - ось симметрии ракетного двигателя), $(+z)$ - крайняя положительная координата по оси $O Z$.

На рис. 2 показано изменение во времени давления продуктов сгорания в камере сгорания ракетного двигателя. На рис. 2, $a$ дано изменение во времени давления в районе переднего днища (место установки воспламенительного устройства). На рис. 2, $б$ представлено изменение во времени давления в районе заднего днища. При срабатывании воспламенительного устройства в камере сгорания (в районе его установки) наблюдается кратковременный локальный интенсивный всплеск давления. В объеме камеры сгорания отслеживается затухающий волновой процесс с постепенным общим подъемом уровня давления за счет также постепенного прогрева и зажигания поверхности горения заряда твердого топлива. При достижении уровня давления, соответствующего некоторому критическому значению (так называемому давлению страгивания, при $t \sim 0,408)$, вскрываются заглушки сопловых блоков. Давление в этот момент в камере сгорания резко падает. За счет интенсивного зажигания поверхности горения заряда твердого топлива (вследствие достаточно высокого уровня давления и особенно больших скоростей потоков газов, обдувающих и прогревающих поверхность горения) давление в камере сгорания резко возрастает и достигает уровня рабочего значения. 



Рис. 2. Изменение во времени давления в камере сгорания: $a$ - в районе переднего днища; $\sigma$ - в районе заднего днища

Примерно такой же процесс протекает и в боковых газоходах ракетного двигателя (рис. 3). На рис. 3, а показано изменение во времени давления продуктов сгорания в вершине первого бокового газохода. На рис. $3, \sigma$ дано изменение во времени давления продуктов сгорания в вершине второго бокового газохода. Здесь, естественно, начального всплеска давления нет (см. рис. 2, a), но переходный затухающий волновой процесс от срабатывания воспламенительного устройства присутствует. Кроме того, здесь более ярко выражен волновой эффект от вскрытия заглушек соплового блока (при $t \sim 0,408$ ). Более того, дальнейшее интенсивное нарастание давления в боковых газоходах сопровождается генерацией колебательного процесса, причем в каждом газоходе этот процесс реализуется по-своему.

Рассмотренный вариант РДТТ особой компоновочной схемы (см. рис. 1) в силу своих функциональных и конструктивных особенностей (по базовым исходным параметрам) имеет весьма значительное время выхода на режим работы (см. рис. 2, 3), что существенно ухудшает его эксплуатационные характеристики. В рамках данной работы была предпринята попытка оптимизации компоновочной схемы данного ракетного двигателя с целью уменьшения его времени выхода на режим работы. Оптимизации были подвергнуты конструкция воспламенительного устройства и геометрическая форма канала заряда твердого топлива РДТТ. На рис. 4 показано изменение во времени давления в камере сгорания в районе переднего днища при различных вариантах конструкции РДТТ. Вариант 1 - исходный (базовый). Вариант 2 - исходный с учетом изменения конструкции воспламенительного устройства. Вариант 3 - исходный с учетом 
изменения конструкции воспламенительного устройства и изменения геометрической формы канала заряда твердого топлива. Из рисунка видно, что предлагаемые технические мероприятия весьма эффективны, причем технологически они достаточно просты в реализации. Время выхода ракетного двигателя на режим работы уменьшено в несколько раз.

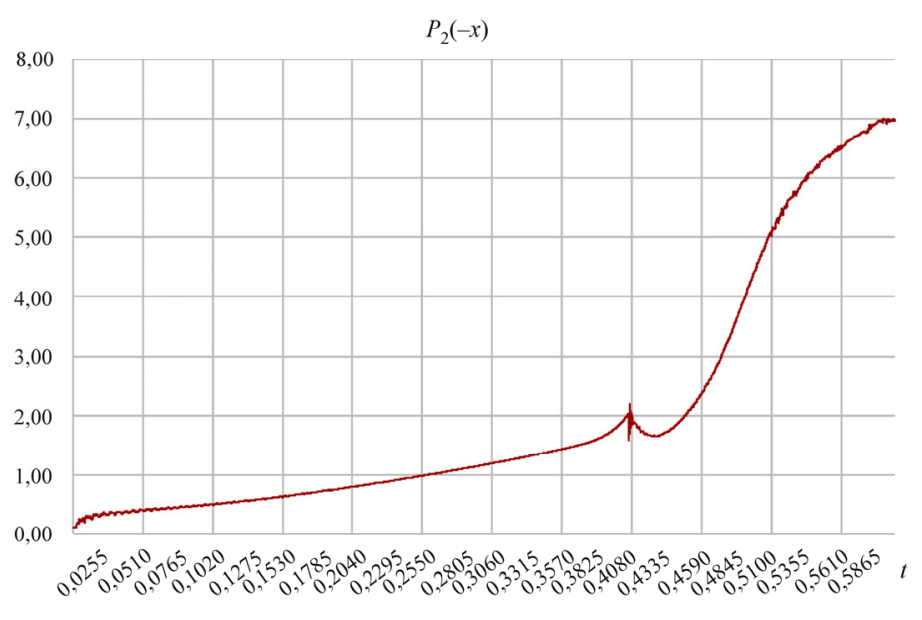

$a$

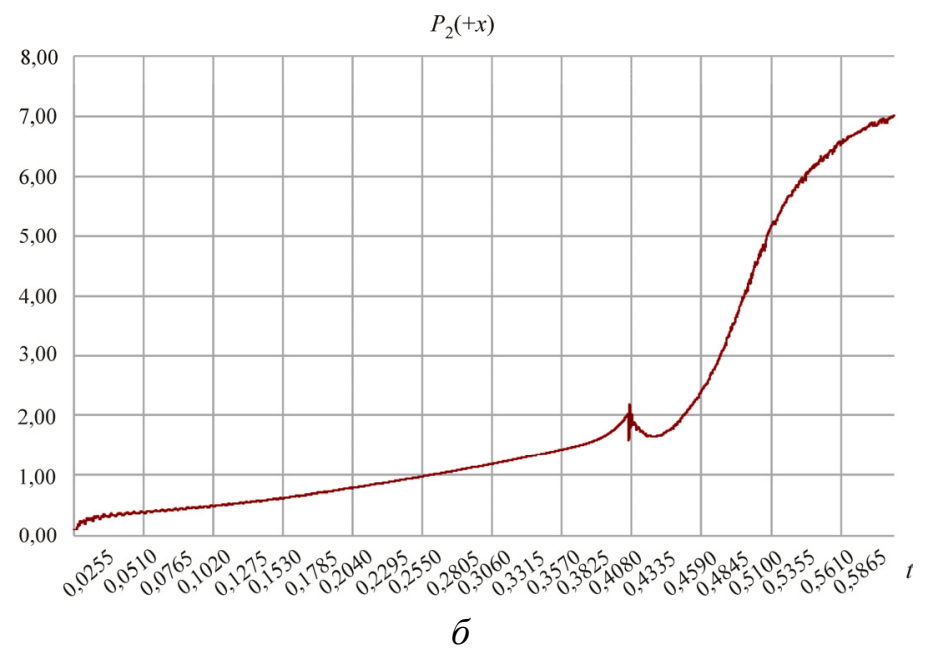

Рис. 3. Изменение во времени давления в вершине: $a$ - первого бокового газохода; $\sigma$ - второго бокового газохода



Рис. 4. Изменение во времени давления в камере сгорания в районе переднего днища при различных вариантах доработки конструкции РДТТ: $1-P_{1}(-z) 3 \mathrm{~B} ; 2-P_{1}(-z) 2 \mathrm{~B} ; 3-P_{1}(-z) 1$ в 
На рис. 5 показано распределение в фиксированный момент времени $(t=0,600)$ по пространству камеры сгорания, боковых газоходов и сопловых блоков РДТТ показателя адиабаты смеси газообразных продуктов сгорания. Распределение дано по сечениям: в плоскости XOZ вдоль оси симметрии ракетного двигателя (см. рис. 5, a), в плоскости $Y O Z$ вдоль оси симметрии ракетного двигателя (см. рис. 5, б), в плоскости XOY в районе размещения канально-щелевого заряда твердого топлива (см. рис. 5, в), в плоскости XOY вдоль оси симметрии боковых газоходов (см. рис. 5, 2). Распределение компонентов продуктов сгорания (точнее, газовой смеси воздуха, продуктов сгорания навески воспламенительного состава и заряда твердого топлива) по пространству камеры сгорания, боковых газоходов и сопловых блоков неравномерное. Наибольшая «концентрация» (максимальное значение показателя адиабаты) продуктов сгорания твердого топлива отмечается у поверхности горения заряда твердого топлива. Наименьшая в районе переднего (на некотором удалении от него) и особенно заднего днища и у оси симметрии ракетного двигателя. Полной симметрии по пространству в плоскости $X O Z$ (см. рис. 5, a), в плоскости $Y O Z$ (см. рис. 5, б) и в плоскости $X O Y$ (см. рис. 5, в, г) по данному параметру потока продуктов сгорания (как и по другим параметрам потока, см. ниже по тексту) не наблюдается. В плоскости $X O Y$ в районе размещения канально-щелевого заряда твердого топлива (см. рис. 5, , ) отслеживаются зоны повышенной концентрации продуктов сгорания твердого топлива по радиусу в плоскости щелей заряда (вдоль оси $O Z$ ). Большую концентрацию продуктов сгорания твердого топлива имеют зоны в плоскости щелей заряда, лежащие в плоскости боковых газоходов.



$a$

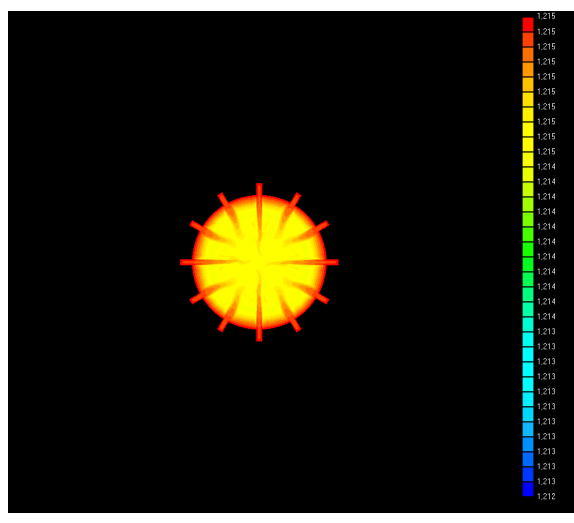

B



$\sigma$

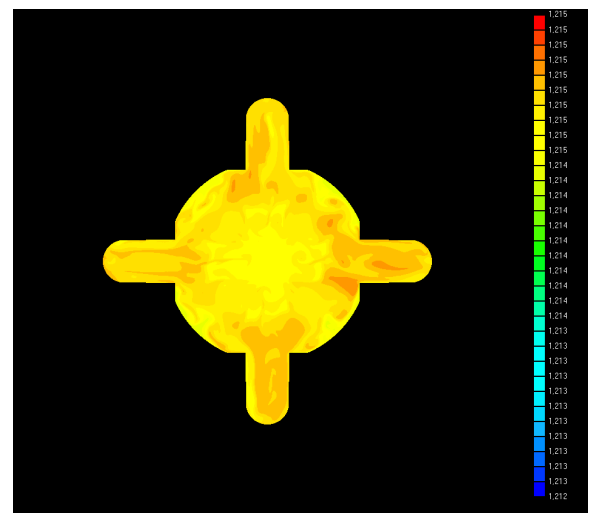

2

Рис. 5. Распределение в фиксированный момент времени $(t=0,600)$ показателя адиабаты продуктов сгорания: $a$-в плоскости XOZ; $\sigma$-в плоскости $Y O Z$; в-в плоскости XOY в районе размещения заряда твердого топлива; 2 - в плоскости $X O Y$ в районе боковых газоходов 
На рис. 6 представлено распределение в фиксированный момент времени $(t=0,600)$ в плоскости $X O Z$ вдоль оси симметрии ракетного двигателя плотности газообразных продуктов сгорания (см. рис. 6, $a$ ) и плотности мелкодисперсных частиц (жидких капель) (см. рис. 6, б). По мере ускорения потока продуктов сгорания, при его движении от камеры сгорания через боковые газоходы в сопловые блоки, плотность продуктов сгорания в целом падает, наиболее существенно в сопловом блоке. Вследствие разворота потока продуктов сгорания в боковом газоходе формируется вихреобразное течение. Особенно это заметно на распределении в этом районе плотности мелкодисперсных частиц (жидких капель). Наибольшая плотность мелкодисперсных частиц образуется на входе в боковой газоход и далее на противоположной к соплу стенке и вершине бокового газохода. Это вихреобразное течение и формирует здесь колебательный процесс (см. рис. 3).

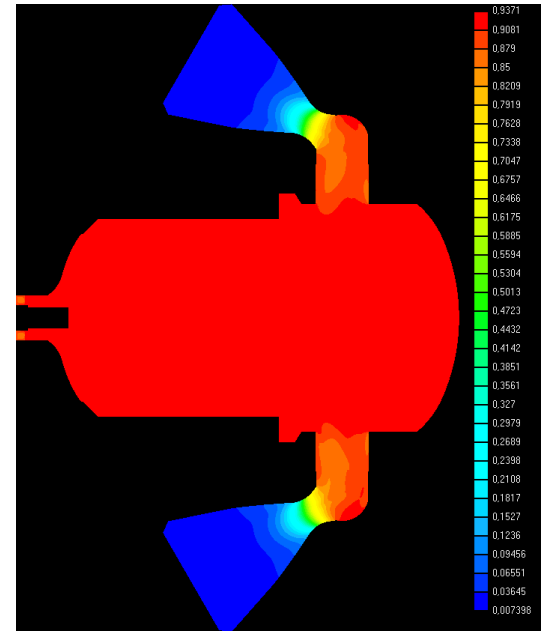

$a$

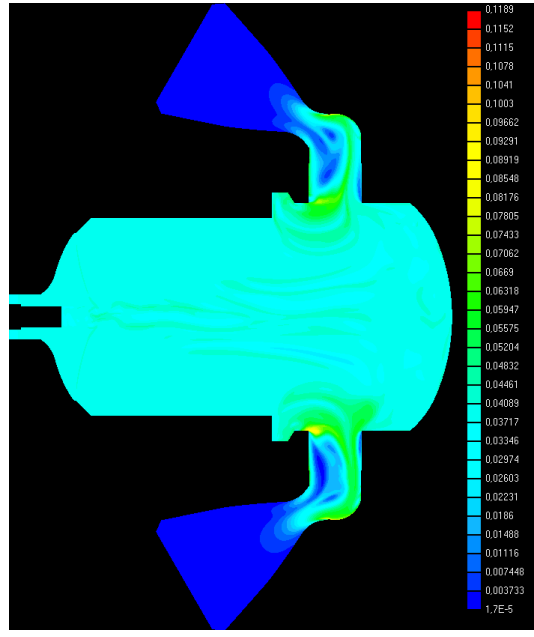

$\sigma$

Рис. 6. Распределение в фиксированный момент времени $(t=0,600)$ : $a$ - в плоскости $X O Z$ плотности газообразных продуктов сгорания; $\sigma$ - в плоскости $X O Z$ плотности мелкодисперсных частиц

На рис. 7-10 показано распределение в фиксированный момент времени $(t=0,600)$ в плоскости $X O Z$ вдоль оси симметрии ракетного двигателя энтропийной функции газовой среды, температуры газообразных продуктов сгорания, скорости звука в газовой среде и модуля вектора скорости газообразных продуктов сгорания соответственно. Данная информация наглядно подтверждает асимметричный по пространству нелинейный вихревой, а следовательно, и волновой характер течения в рассматриваемом РДТТ особой компоновочной схемы.



Рис. 7. Распределение в фиксированный момент времени $(t=0,600)$ в плоскости $X O Z$ энтропийной функции газовой среды



Рис. 8. Распределение в фиксированный момент времени $(t=0,600)$ в плоскости XOZ температуры газообразных продуктов сгорания 


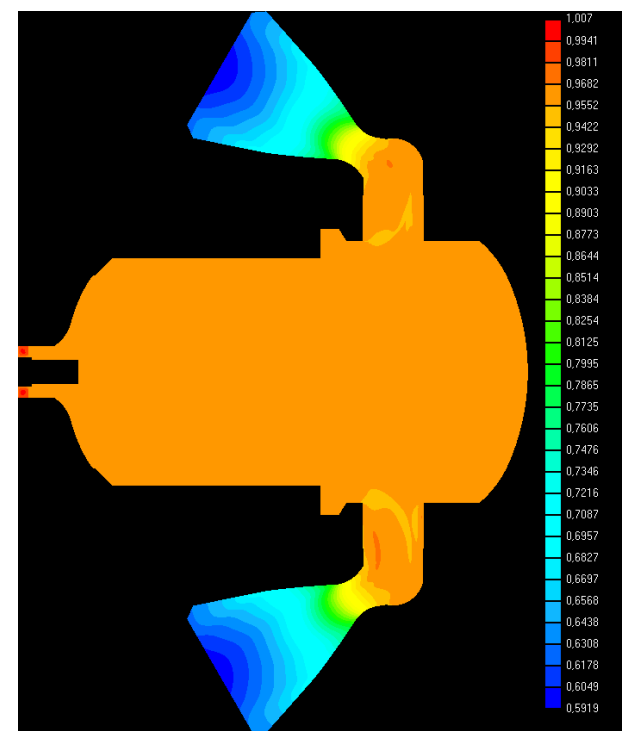

Рис. 9. Распределение в фиксированный момент времени $(t=0,600)$ в плоскости $X O Z$ скорости звука в газовой среде

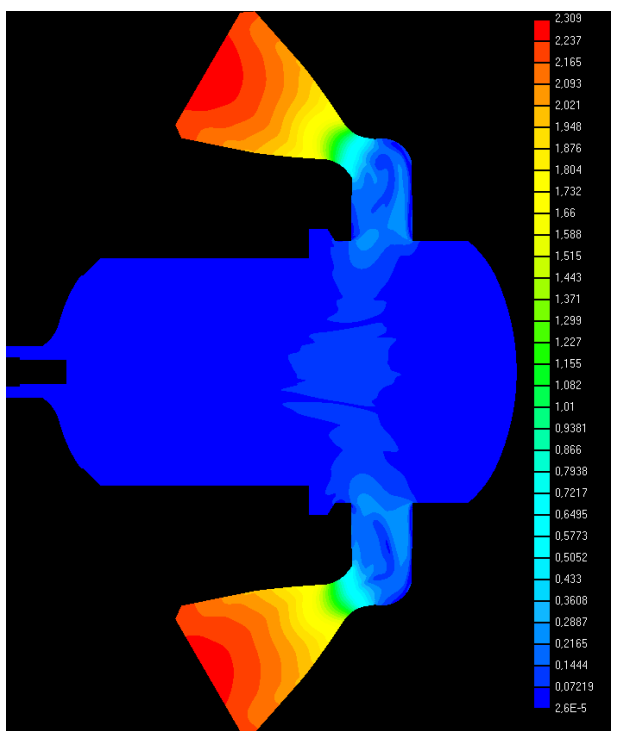

Рис. 10. Распределение в фиксированный момент времени $(t=0,600)$ в плоскости $\mathrm{XOZ}$ модуля вектора скорости газообразных продуктов сгорания

В заключение отметим, что полученная расчетная информация (уровня постановки вычислительного эксперимента) может быть успешно использована при проектировании и отработке новых образцов ракетной техники на твердом топливе с высокими энергомассовыми, прочностными, шумовыми и другими эксплуатационными характеристиками.

\section{Библиографический список}

1. Исследование ракетных двигателей на твердом топливе / под ред. М. Саммерфилда. - М.: ИЛ, 1963. $-440 \mathrm{c}$.

2. Ерохин Б.Т., Липанов А.М. Нестационарные и квазистационарные режимы работы РДТТ. М.: Машиностроение, 1977. - 200 с.

3. Алемасов В.Е., Дрегалин А.Ф., Тишин А.П. Теория ракетных двигателей. - М.: Машиностроение, $1980 .-533 \mathrm{c}$.

4. Гидродинамические источники акустических колебаний в камерах сгорания / В.Ф. Ахмадеев, Г.Н. Гусева, Л.Н. Козлов [и др.] / ЦНИИНТИ КПК. - М., 1990. - 44 с.

5. Численный эксперимент в теории РДТТ / А.М. Липанов, В.П. Бобрышев, А.В. Алиев [и др.]; под ред. А.М. Липанова. - Екатеринбург: УИФ «Наука», 1994. - 301 с.

6. Внутренняя баллистика РДТТ / А.В. Алиев [и др.]; под ред. А.М. Липанова и Ю.М. Милехина; РАРАН. - М.: Машиностроение, 2007. - 504 с.

7. Твердотопливные регулируемые двигательные установки / Ю.С. Соломонов [и др.]; под ред. А.М. Липанова, Ю.С. Соломонова; РАРАН. - М.: Машиностроение, 2011. - 416 с.

8. Yamamoto M., Oguchi H. Gas-particle mixture in a spinning solid rocket motor // ISAS Report. 1983. - No. 607. - P. 1-15.

9. High performance solid rocket motor submerged nozzle/combustion cavity flowfield assessment / J.A. Freeman, J.S. Chan, J.E. Murph, K.E. Xiques // NASA Report. - 1988. - No. CR-179307. - 57 p.

10. Eulerian multi-fluid models for the simulation of dynamics and coalescence of particles in solid propellant combustion / F. Doisneau, F. Laurent, A. Murrone, J. Dupays, M. Massot // Journal of Computational Physics. - 2013. - Vol. 234. - P. 230-262.

11. Егоров М.Ю., Егоров Я.В., Егоров С.М. Исследование неустойчивости рабочего процесса в двухкамерном РДТТ // Известия вузов. Авиационная техника. - 2007. - № 4. - С. 39-43.

12. Егоров М.Ю., Егоров С.М., Егоров Д.М. Численное исследование переходных внутрикамерных процессов при выходе на режим работы РДТТ // Известия вузов. Авиационная техника. - 2010. № 3. - C. 41-45. 
13. Егоров М.Ю., Егоров Д.М. Численное моделирование внутрикамерных процессов в бессопловом РДТТ // XXIII семинар по струйным, отрывным и нестационарным течениям (с междунар. уч.): сб. тр. / под ред. Г.В. Кузнецова, В.Н. Ускова, С.К. Матвеева, В.И. Запрягаева, И.К. Жаровой, Е.Е. Бульбы, Б.В. Борисова, А.В. Захаревича, Е.А. Маслова; Нац. исслед. Том. политехн. ун-т. - 2012. - С. $124-127$.

14. Егоров М.Ю. Численное исследование динамики внутрикамерных процессов при срабатывании специализированного РДТТ // Известия вузов. Авиационная техника. - 2017. - № 4. - С. 104-111.

15. Егоров М.Ю., Егоров Д.М., Егоров С.М. Численное исследование динамики внутрикамерных процессов ракетного двигателя на твердом топливе особой компоновочной схемы. Ч. 1. Постановка вычислительного эксперимента // Вестник Пермского национального исследовательского политехнического университета. Аэрокосмическая техника. - 2018. - № 53. - С. 63-76.

\section{References}

1. M. Summerfield. Issledovaniye raketnykh dvigateley na tverdom toplive [Research of rocket engines on solid fuel]. Moscow: IL, 1963, $440 \mathrm{p}$.

2. Erokhin B.T., Lypanov A.M. Nestatsionarnyye i kvazistatsionarnyye rezhimy raboty RDTT [Nonstationary and quasi-stationary modes of operation]. Moscow: Mashinostroyeniye, 1977, $200 \mathrm{p}$.

3. Alemasov V.E., Drehalyn A.F. Tishin A.P. Teoriya raketnykh dvigateley [Theory of rocket engines]. Moscow: Mashinostroyeniye, 1980, 533 p.

4. Akhmadeev V.F., Guseva G.N., Kozlov L.N. and other. Gidrodinamicheskiye istochniki akusticheskikh kolebaniy $\mathrm{v}$ kamerakh sgoraniya [Hydrodynamic sources of acoustic oscillations in combustion chambers]. Moscow: TSNIINTI KPK, 1990, 44 p.

5. Lypanov A.M, Bobrishev V.P., Alyev A.V. and all. Chislennyy eksperiment v teorii RDTT [Numerical experiment in the theory of solid propellant rocket motors]. Ekaterinburg: UIF «Nauka», 1994, 301 p.

6. Aliyev A. V. Vnutrennyaya ballistika RDTT [Internal ballistics solid propellant motors] ed. by A.M. Lipanov and Y.M. Milekhina. Moscow: Mashinostroyeniye, 2007, 504 p.

7. Solomonov Yu.S., Lipanov A.M. Tverdotoplivnyye reguliruyemyye dvigatelnyye ustanovki [Adjustable solid-fuel propulsion systems]. Moscow.: Mashinostroyeniye, 2011, 416 p.

8. Yamamoto M., Oguchi H. Gas-particle mixture in a spinning solid rocket motor. ISAS Report, 1983, no. 607 , pp. $1-15$.

9. Freeman J.A., Chan J.S., Murph J.E., Xiques K.E. High performance solid rocket motor submerged nozzle/combustion cavity flowfield assessment. NASA Report, 1988, no. CR-179307, 57 p.

10. Doisneau F., Laurent F., Murrone A., Dupays J., Massot M. Eulerian multi-fluid models for the simulation of dynamics and coalescence of particles in solid propellant combustion. Journal of Computational Physics, 2013, Vol. 234, pp. 230-262.

11. Egorov M.Yu., Egorov Ya.V., Egorov S.M. Issledovanie neustoychivosti rabochego protsessa v dvukhkamernom RDTT [Study of working process instability in the two chamber solid propellant rocket engine]. Izvestiya vysshikh uchebnykh zavedeniy. Aviatsionnaya tekhnika, 2007, no. 4, pp. 39-43.

12. Egorov M.Yu., Egorov S.M., Egorov D.M. Chislennoe issledovanie perekhodnykh vnutrikamernykh protsessov pri vykhode na rezhim raboty RDTT [Numerical study of transient interchamber processes when reaching the SPRE]. Izvestiya vysshikh uchebnykh zavedeniy. Aviatsionnaya tekhnika, 2010, no. 3, pp. 41-45.

13. Egorov M.Y., Egorov D.M. Chislennoye modelirovaniye vnutrikamernykh protsessov v bessoplovom RDTT [Numerical Modeling Interchamber processes in at nozzleless solid propellant rocket motors]. XXIII seminar on the jet, tear and unsteady flow (with international participation). Proceedings of National Research Tomsk Polytechnic University, 2012, pp. 124-127.

14. Egorov M.Y. Chislennoe issledovanie dinamiky vnutrikamernykh protsessov pri srabatyvaniy specializirovannogo RDTT [Numerical Research of Intra-Chamber Processes Dynamics during Startup of Special Solid Propellant Engine]. Izvestiya vysshikh uchebnykh zavedeniy. Aviatsionnaya tekhnika, 2017, no. 4, pp. 104-111.

15. Egorov M.Y., Egorov D.M., Egorov S.M. Chislennoye issledovaniye dinamiki vnutrikamernykh protsessov raketnogo dvigatelya na tverdom toplive osoboy komponovochnoy skhemy. Chast 1 . Postanovka vychislitelnogo eksperimenta [Numerical study of the dynamics of in-chamber processes of a rocket engine on solid fuel of a special layout scheme. Part 1. Statement of computational experiment]. PNRPU Aerospace Engineering Bulletin, 2018, no. 53, pp.63-76. 


\section{Об авторах}

Егоров Михаил Юрьевич (Пермь, Россия) - доктор физико-математических наук, профессор, профессор кафедры «Высшая математика» ФПММ» ФГБОУ ВО ПНИПУ (614990, г. Пермь, Комсомольский пр., д. 29, e-mail: egorov-m-j@yandex.ru).

Егоров Дмитрий Михайлович (Пермь, Россия) - кандидат технических наук, зам. главного конструктора Научно-исследовательского института полимерных материалов (614113, г. Пермь, ул. Чистопольская, д. 16, e-mail: egorovdimitriy@mail.ru).

Егоров Сергей Михайлович (Пермь, Россия) - кандидат физико-математических наук, ведущий научный сотрудник Научно-исследовательского института полимерных материалов $(614113$, г. Пермь, ул. Чистопольская, д. 16, e-mail: know_nothing@bk.ru).

Савочкина Анна Александровна (Пермь, Россия) - старший преподаватель кафедры «Высшая математика» ФПММ» ФГБОУ ВО ПНИПУ (614990, г. Пермь, Комсомольский пр., д. 29, e-mail: aidas_76@mail.ru).

\section{About the authors}

Mikhail Yu. Egorov (Perm, Russian Federation) - Doctor of Physical and Mathematical Sciences, Professor, Department of Higher Mathematics, Perm National Research Polytechnic University (29, Komsomolsky av., Perm, 614990, Russian Federation, e-mail: egorov-m-j@yandex.ru).

Dmitry M. Egorov (Perm, Russian Federation) - CSc of Technical Sciences, Deputy Leading Engineer, Research Institute of Polymeric Materials (16, Chistopolskaya st., Perm, 614113, Russian Federation, e-mail: egorovdimitriy@mail.ru).

Sergey M. Egorov (Perm, Russian Federation) - CSc of Physical and Mathematical Sciences, Chief Researcher, Research Institute of Polymeric Materials (16, Chistopolskaya st., Perm, 614113, Russian Federation, e-mail: know_nothing@bk.ru).

Anna A. Savochkina (Perm, Russian Federation) - Senior Lecturer, Department of Higher Mathematics, Perm National Research Polytechnic University (29, Komsomolsky av., Perm, 614990, Russian Federation, e-mail: aidas_76@mail.ru).

Получено 24.07.2018 